\title{
The observational legacy of preon stars - probing new physics beyond the LHC
}

\author{
F. Sandin and J. Hansson \\ Department of Physics, Luleå University of Technology, SE-97187 Luleå, Sweden
}

\begin{abstract}
We discuss possible ways to observationally detect the superdense cosmic objects composed of hypothetical sub-constituent fermions beneath the quark/lepton level, recently proposed by us. The characteristic mass and size of such objects depend on the compositeness scale, and their huge density cannot arise within a context of quarks and leptons alone. Their eventual observation would therefore be a direct vindication of physics beyond the standard model of particle physics, possibly far beyond the reach of the Large Hadron Collider (LHC), in a relatively simple and inexpensive manner. If relic objects of this type exist, they can possibly be detected by present and future x-ray observatories, high-frequency gravitational wave detectors, and seismological detectors. To have a realistic detection rate, i.e., to be observable, they must necessarily constitute a significant fraction of cold dark matter.

PACS numbers: 12.60.Rc, 04.40.Dg, 95.35.+d
\end{abstract}

\section{INTRODUCTION}

It is often assumed that cold dark matter $(\mathrm{CDM})$ is some "exotic" type of weakly interacting elementary particles, primordial relics created in the early universe, not yet detected in particle accelerator experiments. This hypothesis works well in cosmology, but both astrophysical observations, and discrepancies between simulations and observations of galaxies suggest that such a picture may be oversimplified. For example, simulated density profiles of CDM halos are too cuspy, more dwarf galaxies should have been observed because the number of halos is expected to be inversely proportional to the mass, and hydrodynamic simulations produce galaxy disks that are too small, with too low angular momenta [1]. Moreover, there is a close relation between the rotation curve shape and luminosity distribution in spiral galaxies, indicating that CDM couples to luminous matter [2], and the core density in spiral galaxies is roughly constant, scaling with the size of the core [3], in conflict with predictions from such models. Further information and more examples can be found in [4] and references therein.

Considering the complexity of galaxies and the overall success of the traditional view [5], i.e., that CDM is composed of stable weakly interacting (massive) particles (WIMPs), there are no truly compelling reasons to abandon it. It is sensible, however, to also explore alternative possibilities. In particular, since there are indications that CDM couples to baryons, parsimony ("Occam's razor") suggests that it could be a novel state of "ordinary" matter, which decoupled from the radiation in the early universe before the onset of primordial nucleosynthesis. Any structure created at such an early epoch would necessarily have a low characteristic mass and could therefore have remained unnoticed.

The spirit of this idea is not new. Already in the 1980s it was suggested that lumps of stable quark mat-

*Electronic address: Fredrik.Sandin@gmail.com ter, so-called quark nuggets, could have formed in the early universe [6]. Should they exist, such objects contribute to CDM and if they were produced in abundance they could explain some observations that are inconsistent with the traditional view [4]. No observation precludes the possibility that such objects compose the bulk of CDM, provided that the mass of the objects does not exceed $\sim 10^{23} \mathrm{~kg}[4,7]$. This idea has a natural extension to particle scales beneath the quark/lepton level. Subquark particles (hereafter called preons) are motivated in part by the existence of three fermion generations, and other unexplained relations in the standard model of particle physics (SM), which indicate that quarks and leptons could well be composite. Detailed motivations can be found in, e.g., [8] and references therein. If preons exist, stable compact objects ("preon stars") with densities at least ten orders of magnitude higher than in quark nuggets/stars could exist [9, 10]. See also [11] and [12, 13]. While the microscopic motivation for such objects is still somewhat schematic, and the possibility that they formed in the early universe uncertain, it is by no means impossible [11, 14]. As the consequences of their eventual existence are very interesting and farreaching, an investigation of their phenomenology seems well-motivated. In the present paper, we briefly discuss some possibilities to observe compact preon dark matter (CPDM), i.e., relic preon stars/nuggets, and how the quark compositeness scale may be linked to astrophysical data. A different scenario where dark matter is related to preons has been suggested in [15].

\section{PROPERTIES AND FORMATION}

In the mid 1960s it was shown that for solutions to the stellar structure equations, whether Newtonian or relativistic, there is a change in stability whenever the mass reaches an extremum as a function of the central density [16]. The instability in-between white dwarfs and neutron stars, which spans several orders of magnitude of central densities, is an example of this property. Con- 
sequently, beyond the density of the maximum mass neutron (or quark/hybrid) star, $\sim 10^{16} \mathrm{~g} / \mathrm{cm}^{3}$ [17], configurations are unstable. The order of magnitude for this limiting density is valid also for a hypothetical third class of compact stars [18, 19, 20] and for stars composed of exotic hadron/quark condensates. The instability is therefore generally assumed not to end before the Planck scale, if at all. This assumption, however, is valid only in the context of the SM, where quarks and leptons are elementary. If there is at least one deeper layer of constituents, beneath the particles of the SM, a corresponding class of stable compact objects could exist [9, 10, 11]. The density of such objects cannot be explained within the context of the SM. This "window of opportunity" to new physics is our main motivation for investigating means to observe them. In the following, we briefly describe the relation between the compositeness scale and the properties of such objects.

The characteristic density, size, and mass of a compact object depend on the strength of the interactions between the constituent particles, see, e.g., 21]. Qualitatively, the relation between these quantities can be obtained in a simple way. Under the assumption that the equation of state of matter is everywhere causal it follows that the radius, $R$, of a stable compact object must exceed $4 / 3$ of its Schwarzschild radius, $R_{S}=2 G M / c^{2}$, where $M$ is the mass of the object (without the assumption of causality the factor is not $4 / 3$ but $9 / 8$ ), a result that follows from the general relativistic stellar structure equations. Simplifying the density to be constant within the object, this leads to an order of magnitude estimate for the relation between the density, $\rho$, and the mass/radius of the maximum mass configuration

$$
\begin{aligned}
M & \sim \frac{9 c^{3}}{64} \sqrt{\frac{2}{\pi G^{3} \rho}} \\
R & \sim \frac{3 c}{8} \sqrt{\frac{2}{\pi G \rho}} .
\end{aligned}
$$

For neutron stars with $\rho \sim 10^{15} \mathrm{~g} / \mathrm{cm}^{3}$, this estimate yields $M \sim 3 \mathrm{M}_{\odot}$ and $R \sim 10 \mathrm{~km}$, correct order of magnitudes for neutron stars. We assume that the SM is reliable at least up to densities above the onset of the heaviest quark (top), which is of the order $\sim 10^{27} \mathrm{~g} / \mathrm{cm}^{3}$ for a charge-neutral fermion gas of six massive quarks and three massive leptons with an MIT bag constant chosen around the traditional value, $B^{1 / 4} \sim 150 \mathrm{MeV}$. The large mass of the top has been assumed to be a consequence of weak binding between preons, see, e.g., [22]. The phase where preons in the top quark can become deconfined should then have a characteristic density

$$
\rho \sim \frac{m_{t}}{4 / 3 \pi(\hbar c / \Lambda)^{3}} \simeq 9.5 \times 10^{27} \mathrm{~g} / \mathrm{cm}^{3}\left(\frac{\Lambda}{\mathrm{TeV}}\right)^{3},
$$

where $m_{t}$ is the mass of the top quark, $\hbar c / \Lambda$ its "size", and $\Lambda$ is expected to be of the order of the binding force scale parameter, i.e., $\Lambda$ gives the compositeness energy scale. Inserting this estimate in the expressions for the mass and radius of the maximum mass configuration we obtain

$$
\begin{aligned}
M & \sim \frac{3}{32} \sqrt{\frac{6 \hbar^{3} c^{9}}{G^{3} \Lambda^{3} m_{t}}} \simeq 2 \times 10^{24} \mathrm{~kg}\left(\frac{\mathrm{TeV}}{\Lambda}\right)^{3 / 2} \\
R & \sim \frac{1}{4} \sqrt{\frac{6 \hbar^{3} c^{5}}{G \Lambda^{3} m_{t}}} \simeq 3 \times 10^{-3} \mathrm{~m}\left(\frac{\mathrm{TeV}}{\Lambda}\right)^{3 / 2}
\end{aligned}
$$

Other estimates provided in [9, 10, 11] yield slightly different but qualitatively similar results.

CPDM objects could have been created in a first-order phase transition in the early universe [10, 11], by a mechanism similar to that described in [6]. Under rather general assumptions, this scenario requires that the number of microscopic degrees of freedom is higher during the preon era than during the QCD/quark era [14]. This, perhaps counter-intuitive condition is satisfied by some preon models and can be motivated by the simplicity of the representations and the group structure, rather than an economic number of preons. We do not further speculate about the details of the hypothetical phase transition and the process of CPDM formation, as the main aim here is to explore the possibility to detect such objects, if they exist. We therefore assume that there was a firstorder transition from a preon phase to the quark/lepton phase, and that stable preon bubbles formed. What would the characteristic mass of such bubbles be? The density of the radiation background is

$$
\rho_{R} \simeq g_{\text {eff }} \frac{\pi^{2}}{30} \frac{\left(k_{B} T\right)^{4}}{\hbar^{3} c^{5}}
$$

where $g_{\text {eff }}$ is the effective number of microscopic degrees of freedom at temperature $T$. Inserting (6) in Friedmann's equations for a flat universe (the curvature contribution anyway being negligible at early times) we get an expression for the Hubble expansion parameter

$$
H \simeq\left[\frac{8 \pi^{3} G}{90 \hbar^{3} c^{5}} g_{\mathrm{eff}}\right]^{1 / 2}\left(k_{B} T\right)^{2} .
$$

The maximum size of bubbles is limited by the event horizon, i.e., the Hubble radius, $c / H$, at the temperature of the phase transition, $T \simeq \Lambda / k_{B}$. The corresponding maximum mass of a preon bubble is

$$
M_{H} \simeq \frac{4 \pi}{3}\left(\frac{c}{H}\right)^{3} \rho_{R} \simeq 1.0 \times 10^{24} \mathrm{~kg} g_{\mathrm{eff}}^{-1 / 2}\left(\frac{\mathrm{TeV}}{\Lambda}\right)^{2}
$$

which is less than the maximum mass for stable objects (4). From an observational point of view, $M_{H}$ is an estimate for the maximal mass of CPDM objects, because the number of coalescence events during the lifetime of the universe is negligible, e.g., from (15). In reality, a typical preon bubble could be smaller or larger than the Hubble radius at the critical temperature, depending on the details of the phase transition and the bubble dynamics. See [6] for a general discussion about formation 
and evolution of quark bubbles in the QCD phase transition, and [11] for an analogous discussion about preon bubbles. See also 23] and 24], where different scenarios are discussed, leading to maximum masses of quark bubbles that are, respectively, significantly smaller and larger than the Hubble radius at the critical temperature. For example, in 223] it is suggested that the bubbles should be smaller than the Hubble radius by a factor of at least $\ln \left[\left(\hbar c^{5} / G\right)^{1 / 2} /\left(k_{B} T_{\mathrm{QCD}}\right)\right] / 4$, which is about one order of magnitude for $T_{\mathrm{QCD}} \sim 150 \mathrm{MeV}$. A more precise estimate for the maximum mass of CPDM objects would require further assumptions about the nature of preons and their interactions, which are beyond scope of the present paper. In Fig. 1 the estimates for the theoretical maximum mass (4) and the Hubble mass, $M_{H}$ (8), are plotted vs. the compositeness scale, $\Lambda$. Included in the plot are

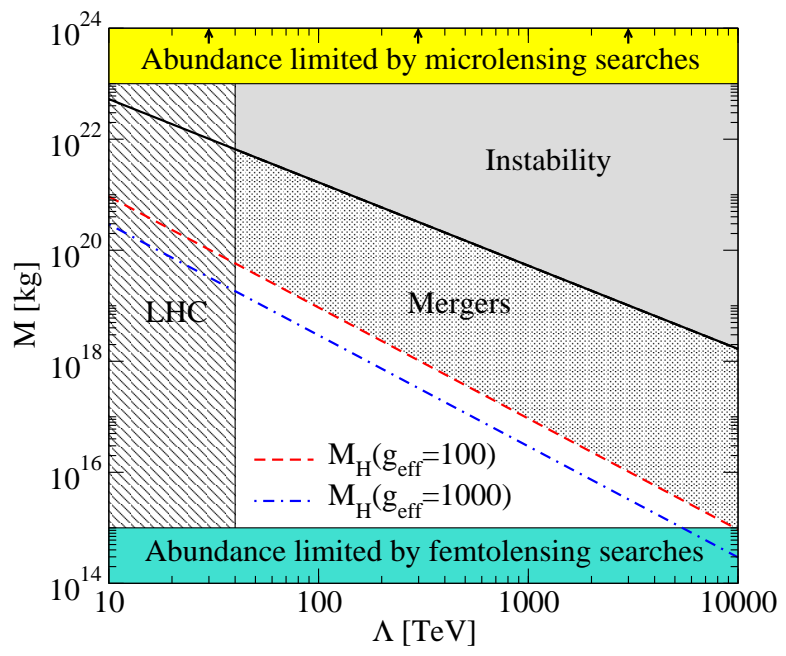

FIG. 1: Constraints on the mass of compact preon dark matter (CPDM) objects vs. the compositeness energy scale, $\Lambda$, which is related to the length-scale of a composite top quark by $\hbar c / \Lambda$. The estimate for the maximum mass of objects formed in the early universe, $M_{H}\left(\Lambda, g_{\text {eff }}\right)$, is the mass within the horizon at the time of the preon phase transition, where $g_{\text {eff }}$ is the effective number of degrees of freedom in the preon phase. The LHC will probe compositeness scales up to about $40 \mathrm{TeV}$. The maximal mass of unobserved compact dark matter objects is $\sim 10^{23} \mathrm{~kg}$ and femtolensing searches rule out $\sim 10^{14}<M<10^{15} \mathrm{~kg}$. No observational technique can presently resolve objects with masses below $10^{14} \mathrm{~kg}$. See the text for details.

also the constraints on the mass of compact CDM from gravitational lensing searches, see the next section. The Large Hadron Collider (LHC) should allow exploration of compositeness scales up to about $\Lambda \sim 40 \mathrm{TeV}$, see [25], where future luminosity upgrades of LHC are discussed also.

\section{GRAVITATIONAL LENSING}

Gravitational lensing is today a well established field of astronomy, with a variety of astrophysical and cosmological applications. Among the many interesting lensing phenomena, there is a possibility to observe low-mass lenses by measuring interference effects between lensed images of narrow astrophysical sources. For lenses with masses in the range $\sim 10^{14} \mathrm{~kg}<M<10^{17} \mathrm{~kg}$, the time delay induced by the lens would be comparable to the oscillation period of a gamma-ray. It has therefore been suggested 26] that lenses with masses in this range could be observed by gravitational lensing of gamma-ray bursts (GRBs). Because the separation of the images would be in the femto-arcsecond range for lenses and sources at cosmological distances, this phenomenon is called "femtolensing". Femtolenses would produce a characteristic pattern in the spectrum of GRBs [27], which is stable on time scales of $1 \mathrm{~s}$, but might slowly drift on time scales of $10 \mathrm{~s}$ due to the relative motion of the lens and source.

No evidence for the existence of femtolenses presently exist, but a number of GRB spectra, see [28] and references therein, have significant features that yet remain to be explained and are similar [29] to those in a femtolensing model spectrum. In particular, the GRB detector aboard the Ginga spacecraft recorded "absorption" features with credible significance near 20 and $40 \mathrm{keV}$, especially for the burst GRB 880205 [30] and somewhat less convincingly in the burst GRB 870303 [31]. These features were originally interpreted as evidence for cyclotron scattering of electrons in a strong magnetic field and, as a consequence, a galactic origin of some GRBs, see, e.g., 28, 32]. More recent observations (afterglows, supernova-GRB connection, etc.) and theoretical models of GRBs falsify this explanation, in particular because these were long GRBs, known to occur at cosmological distances. The origin of the features observed with Ginga is therefore an unsolved mystery. Similar features in the spectra of GRBs have been detected in a number of other missions, notably at 11 and $35 \mathrm{keV}$ in GRB 890306 by Lilas [33], and at 50 and $70 \mathrm{keV}$ in the two peaks of GRB 780325 by HEAO A-4 34]. Similar features have been detected also by the BATSE spectroscopy detectors, see [35] and references therein.

For more massive lenses, the energy-dependent spectra from a single GRB detector provide no useful information. Instead, the spatial interference effect needs to be measured. Two spacecrafts separated by a distance that exceeds the radius of the Einstein ring of the lens, $R_{E} \sim \sqrt{G M /(H c)} \sim 10^{7} \mathrm{~m} \times \sqrt{M /\left(10^{15} \mathrm{~kg}\right)}$, could detect lenses with masses in the range $\sim 10^{15} \mathrm{~kg}<M<$ $10^{23} \mathrm{~kg}$ [36]. No present result limits the amount of CPDM with masses in this range 37]. Consequently, refined femto- and picolensing searches could be used to detect CPDM with masses in the range $10^{14} \mathrm{~kg}<M<$ $10^{23} \mathrm{~kg}$. A large abundance of CPDM with $M>10^{23} \mathrm{~kg}$ is, however, not consistent with observations [7]. This does not preclude the possibility that a small fraction of 
CDM is in that form, but since the corresponding compositeness scale is within reach of the LHC, see Fig. 1, there is no reason to discuss that possibility here. In the following, we briefly discuss the femtolensing effect on the spectrum of GRBs.

The magnification functions for point and extended sources have been derived in [27]. These functions are not trivial to obtain and have to be calculated numerically. We have therefore provided an on-line tool [38] for calculation of femtolensing magnification functions and model GRB spectra, which implements the model in 27] with some extensions. The magnification function depends on four parameters, the mass and redshift of the lens, the angular separation of the source and lens, and the angular width of the source. The width of the lens is neglected, because it has practically no effect as long as the lenses are smaller than their Einstein ring. We denote the angular diameter distances of the lens and the source from the observer, and of the source from the lens with $d_{L}, d_{S}$, and $d_{L S}$, respectively. The distance, $r_{s}$, between the source and the optical axis is measured in the dimensionless quantity

$$
r_{s}=\frac{\sqrt{\xi^{2}+\eta^{2}}}{d_{s} \theta_{E}}
$$

where $\theta_{E}=\sqrt{4 G M d_{L S} /\left(c^{2} d_{L} d_{S}\right)}$ is the angular radius of the Einstein ring and $(\xi, \eta)$ are the Cartesian coordinates of the source in the source plane. The dimensionless width of the source, $\sigma_{s}$, is defined analogous to $r_{s}$, i.e., the actual width is divided by $d_{s} \theta_{E}$. Some femtolensing spectra are plotted in Fig. 2, for three different widths of a GRB, which is assumed to have a fixed position relative to the optical axis, $r_{s}=0.5$. The model spectrum of the

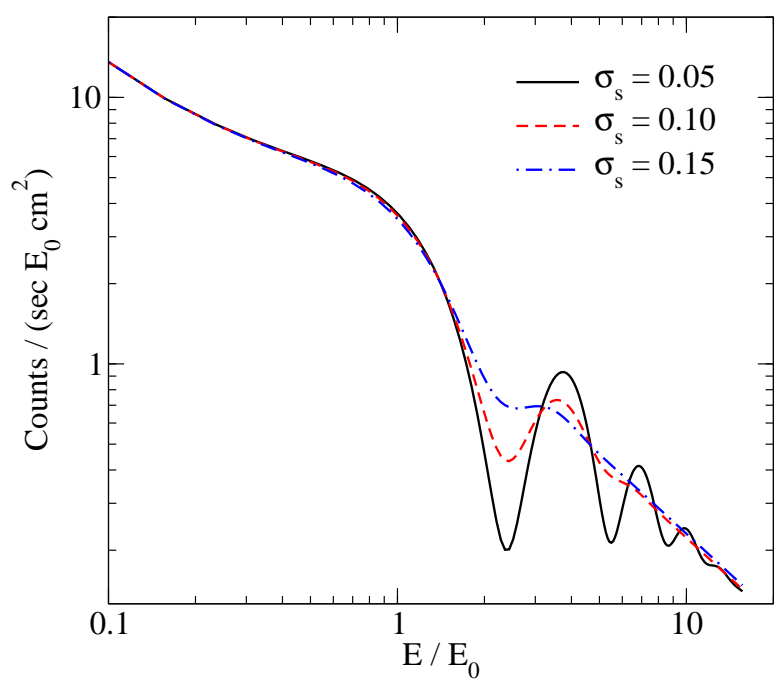

FIG. 2: Femtolensing of a gamma-ray burst (GRB) with model spectrum $\left(E / E_{0}\right)^{-1}$, for three different widths of the source, $\sigma_{s}$. The GRB has a fixed position relative to the optical axis, $r_{s}=0.5$, see text. These spectra were calculated with the on-line interface [38].

GRB is a assumed to be a power law, with an exponent of -1 . The energy scale depends on the redshift, $z$, and mass, $M$, of the lens according to

$$
\begin{aligned}
E_{0} & =\frac{h c^{3}}{4 \pi G M(1+z)} \\
& \simeq 1.3 \times 10^{3} \mathrm{keV}\left(\frac{10^{14} \mathrm{~kg}}{M}\right)\left(\frac{1}{1+z}\right),
\end{aligned}
$$

for any model spectrum of the GRB. In Fig. 3 a femtolensing spectra is superimposed on the spectral data of GRB 880205 for power law models of the GRB spectrum.

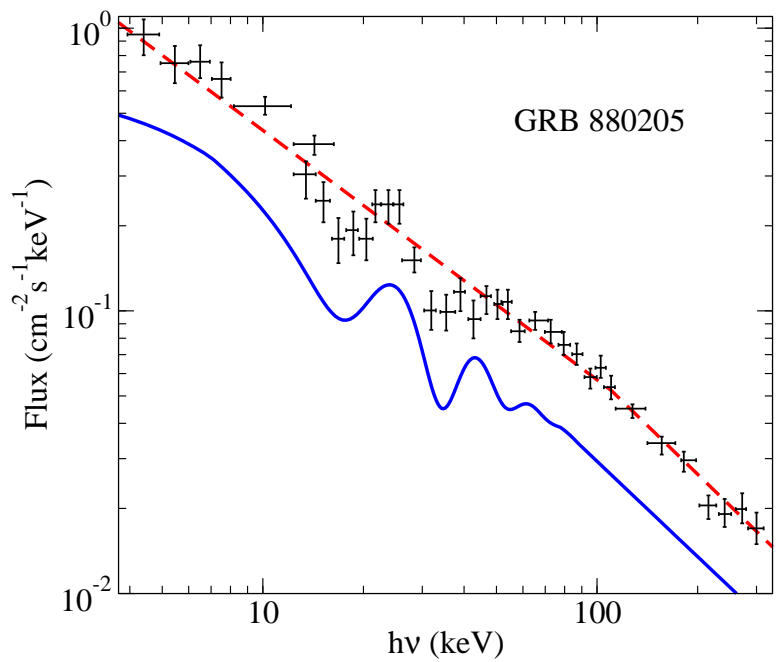

FIG. 3: Ginga spectral data of GRB 880205 for a power law model of the incoming spectrum (dashed line), which is ruled out at more than $99.99 \%$ confidence level [28]. The observed spectrum has line features at $h \nu \simeq 20$ and $40 \mathrm{keV}$, which could be due to gravitational lensing (diffraction) of a Gaussian source by a $\sim 10^{16} \mathrm{~kg}$ object at redshift $z \sim 1$ (solid line). The spectral data depend on the model used and should not be directly compared to the diffraction spectrum, which therefore has been shifted downwards to enhance viewing [29]. The main concern here is the location of the line features.

Because the amplitude of the femtolensing magnification function decays with frequency (and the width of the source), detectors that have energy thresholds well below the first minima should be used in femtolensing searches. According to Eq. (15) in [27], the first minimum of the magnification function is located at

$$
\begin{aligned}
E_{1} & \simeq \frac{3 \pi E_{0}}{8 r_{s}} \\
& \simeq 1.6 \times 10^{3} \mathrm{keV}\left(\frac{10^{14} \mathrm{~kg}}{M}\right)\left(\frac{1}{1+z}\right)\left(\frac{1}{r_{s}}\right) .
\end{aligned}
$$

Spectra from the Transient Gamma-Ray Spectrometer and the BATSE spectroscopy detectors used in recent searches for absorption line features in GRB spectra were limited to $E>40 \mathrm{keV}$ and $20 \mathrm{keV}$, respectively, see [35, 39] and references therein. Consequently, the advantages of these instruments fall short in searches 
for femtolenses of high mass due to the relatively high lower-energy thresholds. The absorption features in GRB 870303, GRB 880205, and GRB 890306 observed in earlier missions would appear less significant if observed with these instruments. In particular, the low-energy absorption features in these bursts would not be detected. The limit on the abundance of femtolenses given in 37] should therefore not be taken too seriously for more massive femtolenses. For masses in the picolensing range, there are presently no limits on the abundance of CPDM (other than $\rho_{\mathrm{CPDM}} \leq \rho_{\mathrm{CDM}}$ ). A refined search for femto- and picolensing features in high-resolution spectra of GRBs would therefore provide useful constraints on the abundance of CPDM and similar compact dark matter objects.

\section{GRAVITATIONAL WAVES FROM BINARIES}

While gravitational pico- and femtolensing can be used to detect and estimate the mass spectrum of CPDM, these methods provide little information about the actual density of the lenses $\left(R \lesssim d_{L} \theta_{E}\right)$. Consequently, lensing methods alone cannot provide detailed information about the nature of the objects and their constituents. One possibility to constrain the upper limit size of CPDM is to measure high-frequency gravitational wave (GW) radiation emitted from binary systems. In the following, we estimate the properties and expected rate of such events for objects with masses in the range $10^{15} \mathrm{~kg}<M<10^{23} \mathrm{~kg}$, which roughly is the range unconstrained by gravitational lensing searches.

Assuming that the objects are distributed randomly in the solar neighbourhood, the probability distribution function for the semi-major axis, $a$, of binaries is [40]

$$
P(a) d a=\frac{3}{4}\left(\frac{a}{\bar{x}}\right)^{3 / 4} \exp \left[-\left(\frac{a}{\bar{x}}\right)^{3 / 4}\right] \frac{d a}{a},
$$

where $\bar{x}$ is the mean separation. Typically, the tidal forces from nearby objects add angular momentum to a binary and head-on collisions are thereby avoided. We assume that the dark halo density in the solar neighbourhood is $0.0079 \mathrm{M}_{\odot} \mathrm{pc}^{-3}$ [41]. For simplicity, we also assume that the bulk of the dark halo is in the form of CPDM of equal masses. The results can readily be generalised to an arbitrary fraction of CPDM. The mean separation is

$$
\bar{x} \simeq\left(\frac{0.0079 \mathrm{M}_{\odot}}{M}\right)^{-1 / 3} \mathrm{pc} .
$$

The remaining time before coalescence, $\tau$, due to emission of GWs depends on the masses, the semi-major axis, and the eccentricity of the orbit. For small $\tau$ the eccentricity can be neglected, as the radiation reaction acts to reduce it. For a circular orbit, the coalescence time is
42

$$
\tau=\frac{5 c^{5}}{512 G^{3}} \frac{a^{4}}{M^{3}}
$$

The probability distribution function (12) can be expressed in the coalescence time $\tau$. Consequently, the relative number of coalescence events within a time $t$ is obtained by

$$
\begin{aligned}
\int_{0}^{t} P(\tau) d \tau & =1-\exp \left[-\bar{x}^{-3 / 4}(\kappa t)^{3 / 16}\right], \\
\kappa & =\frac{512 G^{3} M^{3}}{5 c^{5}}
\end{aligned}
$$

where $\int_{0}^{\infty} P(\tau) d \tau=1$. The exponent in (15) is small for all masses considered here, at any relevant timescale, $t$. We therefore make the approximation $1-\exp (-x) \simeq x$. The total number of objects, $N(D)$, within a distance $D$ can be expressed in the local dark halo density and the mass of the objects. The number of coalescence events, $N_{c}$, within a time $t$ is $N_{c}=N(D) \int_{0}^{t} P(\tau) d \tau$, which yields

$$
N_{c} \simeq 4.9\left(\frac{D}{\mathrm{pc}}\right)^{3}\left(\frac{10^{15} \mathrm{~kg}}{M}\right)^{11 / 16}\left(\frac{t}{\mathrm{yrs}}\right)^{3 / 16} .
$$

This estimate for the coalescence rate scales linearly with the fraction of CPDM, i.e., there is an extra factor $\rho_{\mathrm{CPDM}} / \rho_{\mathrm{CDM}}$ on the right-hand side of (17). In order to obtain a realistic event rate, a detector sensitive enough to detect CPDM coalescence events at a distance of several pc is needed. Next, we estimate the frequency and amplitude of such events.

The frequency of GWs, $f_{g}$, emitted from a binary in a circular orbit is twice the Kepler frequency

$$
\begin{aligned}
f_{g} & =\frac{1}{\pi}\left(\frac{2 M G}{a^{3}}\right)^{1 / 2} \\
& \simeq 6.0 \times 10^{11} \mathrm{~Hz}\left(\frac{\mathrm{sec}}{\tau}\right)^{3 / 8}\left(\frac{10^{15} \mathrm{~kg}}{M}\right)^{5 / 8}
\end{aligned}
$$

because the waves are essentially generated by the quadrupole moment of the binary. The power emitted in GWs is 42

$$
\begin{aligned}
L_{g} & =\frac{64 G^{4}}{5 c^{5}}\left(\frac{M}{a}\right)^{5} \\
& \simeq 1.4 \times 10^{16} \mathrm{~W}\left(\frac{M}{10^{15} \mathrm{~kg}} \frac{f_{g}}{\mathrm{GHz}}\right)^{10 / 3},
\end{aligned}
$$

and the amplitude of the GWs at a distance $D$ from the source is

$$
\begin{aligned}
h & =\left(\frac{G L_{g}}{\pi^{2} c^{3}}\right)^{1 / 2} \frac{1}{f_{g} D} \\
& \simeq 1.9 \times 10^{-36}\left(\frac{M}{10^{15} \mathrm{~kg}}\right)^{5 / 3}\left(\frac{f_{g}}{\mathrm{GHz}}\right)^{2 / 3}\left(\frac{\mathrm{pc}}{D}\right)(.20)
\end{aligned}
$$


The frequency dependent amplitude (20) is plotted in Fig. 4 for different masses, $M$, and distances, $D$, chosen such that 10 coalescence events per year are expected with at least that amplitude (if the CPDM fraction of CDM is less than one, the number of events per year is lowered by the same factor). Also indicated

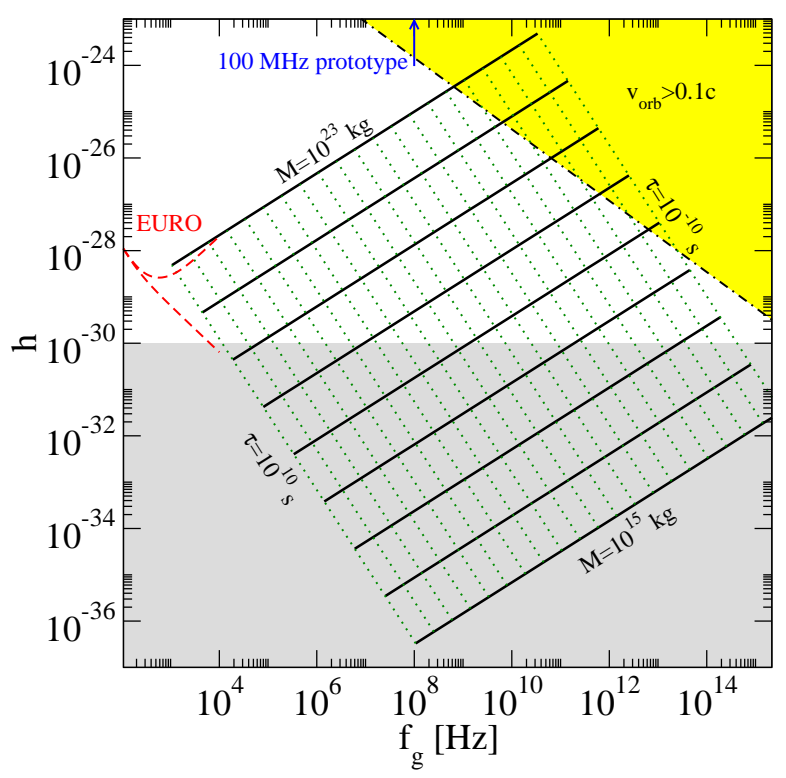

FIG. 4: Amplitude vs. frequency for gravitational waves emitted from an equal-mass binary system in circular orbit. The distance is such that 10 coalescence events per year is expected within that range, i.e., $N_{c}=10$ in (17). The solid lines denote the frequency-amplitude relation for different masses, $M$, of the CPDM objects, in steps of one order of magnitude. The coalescence time (14) is denoted by the dotted lines, also in steps of one order of magnitude. The dash-dotted line corresponds to an orbital velocity of $10 \%$ of the speed of light. Dashed lines denote the lower sensitivity curves for an observational time of 5 years with EURO, according to two different design specifications (shot-noise limited antenna with a kneefrequency of $1000 \mathrm{~Hz}$ and a xylophone-type interferometer). The sensitivity of the first prototype $100 \mathrm{MHz}$ detector in the UK [44] is presently insufficient to detect CPDM coalescence events. The shaded region, $h<10^{-30}$, apparently is beyond reach of experiments and could be polluted by the relic gravitational wave background, see [43] and references therein.

in the plot are the coalescence time (14), the threshold of the relativistic domain, where the orbital velocity, $v_{\text {orb }}=c\left(R_{S} / a\right)^{1 / 2}$, is $10 \%$ of the speed of light, and an estimate for the sensitivity of future detectors, $h_{\min } \sim 10^{-30}$, see [43] and references therein. Even if this estimate for the sensitivity could be exceeded, coalescence events with significantly lower amplitudes would be difficult to distinguish from the stochastic GW background, created by quantum fluctuations in the early universe. This background exists in most popular cosmological models and, due to the expansion of the universe, the amplitudes of the initial fluctuations are amplified and should approach the $h \sim 10^{-30}$ level [43]. Because $h \propto N_{c}^{-1 / 3}$, the amplitudes in Fig. 4 will increase only by a factor two for an order of magnitude decrease of the event rate. We therefore choose $N_{c}=10$, to compensate for the simplifying assumption that $\rho_{\mathrm{CPDM}} / \rho_{\mathrm{CDM}}=1$.

The planned spectral noise density for the European Gravitational Wave Observatory (EURO) in the range $10-10000 \mathrm{~Hz}$ is 45 ]

$$
\begin{aligned}
S_{n}(f)= & 10^{-50}\left[\left(\frac{f}{245 \mathrm{~Hz}}\right)^{-4}+\left(\frac{f}{360 \mathrm{~Hz}}\right)^{-2}\right. \\
& \left.+\left(\frac{f_{k}}{770 \mathrm{~Hz}}\right)\left(1+\frac{f^{2}}{f_{k}^{2}}\right)\right] \mathrm{Hz}^{-1},
\end{aligned}
$$

where $f_{k}=1000 \mathrm{~Hz}$ is the knee frequency. Alternatively, EURO will be based on a xylophone-type interferometer, which has higher sensitivity at high frequencies. The spectral noise density for the latter choice is described by (21) when the last $f_{k}$-dependent term is omitted. The characteristic amplitude of a $\mathrm{GW}$ is $h_{c}=h \sqrt{n}$, where $n=f_{g} \Delta T$ is the number of cycles during an observational time of $\Delta T$. The wave strength of GWs from a monochromatic source observed with an interferometer is $h_{s}=h_{c} / \sqrt{5 f_{g}}$. Consequently, the minimum amplitude, $h_{\text {min }}$, that can be resolved with EURO after an observational time $\Delta T$ is $h_{\min }=\sqrt{5 S_{n}(f) / \Delta T}$. This estimate for the sensitivity of EURO is plotted in Fig. (4) for an observational time of five years. High-mass CPDM is marginally within range of the next generation of gravitational wave detectors. However, the semi-major axis of a binary is

$$
\begin{aligned}
a & =R_{S}\left(\frac{c^{3}}{2 \pi G M f_{g}}\right)^{2 / 3} \\
& \simeq 1.6 \times 10^{7} R_{S}\left(\frac{10^{15} \mathrm{~kg}}{M} \frac{\mathrm{GHz}}{f_{g}}\right)^{2 / 3},
\end{aligned}
$$

so in order to get useful constraints on the compactness of CPDM, a detector sensitive at higher frequencies is needed. Interestingly, high-frequency GW detectors are laboratory-scale devices that are relatively inexpensive to construct. A first $100 \mathrm{MHz}$ prototype has recently been built in the UK 44]. If the sensitivity of such detectors would approach the estimates given in [43], they would provide useful constraints on CPDM. The range $10^{15} \mathrm{~kg}<M \lesssim 10^{18} \mathrm{~kg}$ would, however, only be accessible by rare nearby events.

\section{CONCLUSION \& DISCUSSION}

If quarks and leptons are composite particles, superdense preon stars (or "nuggets") could exist [9, 10, 11]. While the microscopic motivation for such objects is still somewhat schematic and the exact process of formation uncertain, the consequences of their eventual existence are far-reaching. In the present paper we briefly investigate their phenomenology, assuming that they formed in 
the early universe and contribute significantly to CDM. Their maximum mass is roughly limited by the horizon at the time of formation, $M_{H} \sim 10^{24} \mathrm{~kg} g_{\mathrm{eff}}^{-1 / 2}(\mathrm{TeV} / \Lambda)^{2}$, where $\Lambda$ is the quark compositeness energy scale and $g_{\text {eff }}$ is the number of microscopic degrees of freedom in the primordial preon phase. This is a factor $\sim 2 \sqrt{g_{\text {eff }} \Lambda / \mathrm{TeV}}$ lower than the maximum mass for stable hydrostatic configurations. However, the typical mass could be lower or higher than this estimate, depending on the properties of preons and their interactions. Gravitational lensing searches put strong constraints on the abundance of CDM objects with masses in the ranges $M \gtrsim 10^{23} \mathrm{~kg}$ and $10^{14} \lesssim M \lesssim 10^{15} \mathrm{~kg}$. Unexplained features in GRB spectra observed by, e.g., Ginga, Lilas, and HEAO A-4 motivate a continued search for gravitational pico- and femtolenses. This would provide useful constraints on the abundance of compact CDM objects with masses in the range $10^{15} \lesssim M \lesssim 10^{23} \mathrm{~kg}$, corresponding to a maximum compositeness energy scale for CPDM of a few thousand TeV. This observational technique, however, provides little information about the nature of the lenses, because their size and density is limited only by the radius of their Einstein ring. Future high-frequency gravitational wave detectors could provide complementary information about the density of compact CPDM binaries, but it is presently unclear whether it is possible to detect the chirp signal of a low-mass binary as the objects coalesce [46]. This would be necessary in order to obtain a useful constraint on the radii and, consequently, a lower-limit for the density of the objects. In an optimistic scenario, where the mass of the CPDM objects is comparable to $M_{H}$, this method could be useful to indirectly detect compositeness up to a few hundred $\mathrm{TeV}$. Another possibility to detect compact CDM objects and to constrain their density is by seismology, i.e., by measuring the seismic waves generated as they pass through the Earth or the Moon, see [47] and references therein. Unlike the observational methods discussed above, this method is useful in scenarios where the typical mass of the objects is low, as the collision rate increases with the number density of objects. The cross-section of a CPDM object would be at least six orders of magnitude smaller than for a quark nugget of equal mass, making it possible to distinguish them. Should CPDM objects exist, their observational detection may well be the only means, for quite a long time, to discover compositeness beyond the reach of the LHC and other near-future accelerators. As the observational techniques discussed here are useful also for other purposes, and are already in operation to some extent, they constitute a comparatively simple and inexpensive way to test the CPDM hypothesis.

\section{Acknowledgments}

F.S. acknowledges support from the Swedish Graduate School of Space Technology and J.H. acknowledges support from Carl Tryggers stiftelse.
[1] J. P. Ostriker \& P. J. Steinhardt, Science 300, 1909 (2003); J. R. Primack, Nucl. Phys. Proc. Suppl. 124, 3 (2003); New. Astron. Rev. 49, 25 (2005); G. Bertone, D. Hooper \& J. Silk, Phys. Rept. 405, 279 (2005).

[2] R. Sancisi, Proc. IAU Symposium 220, eds. S. D. Ryder, D. J. Pisano, M. A. Walker \& K. C. Freeman, Astron. Soc. Pac. 220 (2004); astro-ph/0311348

[3] G. Gentile, P. Salucci, U. Klein, D. Vergani \& P. Kalberla, Mon. Not. Roy. Astron. Soc. 351, 903 (2004).

[4] A. Zhitnitsky, Phys. Rev. D 74, 043515 (2006).

[5] N. A. Bahcall, J. P. Ostriker, S. Perlmutter \& P. J. Steinhardt, Science 284, 1481 (1999); L. M. Wang, R. R. Caldwell, J. P. Ostriker \& P. J. Steinhardt, Astrophys. J. 530, 17 (2000).

[6] E. Witten, Phys. Rev. D 30, 272 (1984).

[7] R. B. Metcalf \& J. Silk, Phys. Rev. Lett. 98, 071302 (2007).

[8] J-J. Dugne, S. Fredriksson \& J. Hansson, Europhys. Lett. 57, 188 (2002).

[9] J. Hansson \& F. Sandin, Phys. Lett. B 616, 1 (2005).

[10] F. Sandin, Eur. Phys. J. C 40, S15 (2005).

[11] J. E. Horvath, Astrophys. Space Sci. 307, 419 (2007).

[12] H. Knutsen, Gen. Rel. Grav. 23, 843 (1991).

[13] G. H. A. Cole \& J. Dunning-Davies, Gravitation 4, 79 (1999) (in Russian).

[14] H. Nishimura \& Y. Hayashi, Phys. Rev. D 35, 3151 (1987).

[15] V. Burdyuzha, O. Lalakulich, Yu. Ponomarev \& G.
Vereshkov; hep-ph/9907531, astro-ph/9912555

[16] B. K. Harrison, K. S. Thorne, M. Wakano \& J. A. Wheeler, Gravitation Theory and Gravitational Collapse (University of Chicago Press, Chicago, 1965).

[17] C. Kettner et al., Phys. Rev. D 51, 1440 (1995).

[18] U. H. Gerlach, Phys. Rev. 172, 1325 (1968), U. H. Gerlach, Ph.D. thesis, Princeton University, 1968.

[19] N. K. Glendenning \& C. Kettner, Astron. Astrophys. 353, L9 (2000).

[20] K. Schertler et al., Nucl. Phys. A 677, 463 (2000).

[21] G. Narain, J. Schaffner-Bielich \& I. N. Mishustin, Phys. Rev. D, 74, 063003 (2006).

[22] J. C. Pati, Phys. Rev. D 30, 1144 (1984).

[23] C. J. Hogan, Phys. Lett. B 133, 172 (1983).

[24] W. N. Cottingham, D. Kalafatis \& R. Vinh Mau, Phys. Rev. Lett. 73, 1328 (1994).

[25] F. Gianotti, Nucl. Phys. Proc. Suppl., 147, 23 (2005).

[26] A. Gould, Astrophys. J. 386, L5 (1992).

[27] K.Z. Stanek, B. Paczyński \& J. Goodman, Astrophys. J. 413, L7 (1993).

[28] E. E. Fenimore et al., Astrophys. J. 335, L71 (1988).

[29] The observed counts in a gamma-ray detector corresponds to the energy loss spectrum, which is created by the gamma photons as they interact with the medium in the detector, e.g., sodium iodide or germanium. Consequently, it is not certain that the energy of a particular count equals the energy of an incident photon. The spectrum of the light entering the detector can therefore not 
be deduced from data. Instead, model spectra are used in conjunction with data to calculate the probability that the physical spectrum is/is not explained by a particular model. In this procedure, details about the detector response functions and the original data are needed. We were unable to obtain this information for the detector aboard Ginga and for GRB 880205. A formal detailed comparision with femtolensing spectra has therefore not been done.

[30] T. Murakami et al., Nature 335, 234 (1998).

[31] P.E. Freeman et al., Astrophys. J. 524, 753 (1999).

[32] J. C. L. Wang et al., PRL 63, 1550 (1989).

[33] C. Barat, Astron. Astrophys. Suppl. Ser. 97, 43 (1993).

[34] G. J. Hueter, Ph.D. Thesis (University of California, San Diego, 1987).

[35] M. S. Briggs et al., Proc. 3rd INTEGRAL workshop, eds. A. Bazzano, C. Winkler \& G. Palumbo, Astrophys. Lett. Commun. 38/39 (1999); astro-ph/9901224.

[36] R. J. Nemiroff \& A. Gould, Astrophys. J. 452, L111
(1995).

[37] G. F. Marani et al., Astrophys. J. 512, L13 (1999).

[38] On-line femtolensing calculator: http://femtolensing.dyndns.org

[39] P. Kurczynski et al., Astrophys. J. 543, 77 (2000).

[40] K. Ioka et al., Phys. Rev. D 58, 063003 (1998).

[41] C. Alcock et al., Astrophys. J. 542, 281 (2000).

[42] C. W. Misner, K. S. Thorne \& J. A. Wheeler, Gravitation (W. H. Freeman and Company, San Francisco, 1973).

[43] G. S. Bisnovatyi-Kogan \& V. N. Rudenko, Class. Quant. Grav. 21, 3347 (2004).

[44] A. M. Cruise \& R. M. J. Ingley, Class. Quant. Grav. 23, 6185 (2006)

[45] EURO homepage: http://www.astro.cf.ac.uk/geo/euro

[46] A. M. Cruise, private communication.

[47] E. T. Herrin, D. C. Rosenbaum \& V. L. Teplitz, Phys. Rev. D 73, 043511 (2006). 